\title{
The effect of task location and task type on backward inhibition
}

\author{
KATHERINE ARBUTHNOTT \\ University of Regina, Regina, Saskatchewan, Canada
}

\begin{abstract}
Alternating tasks in a sequence of task switches results in impaired performance, relative to switches across three different tasks, an effect known as backward inhibition. Despite the robustness of this effect across task and response variations, backward inhibition is not observed when tasks are uniquely located at different points in space (Arbuthnott, 2005). Three hypotheses about the source of this anomaly were tested. Experiment 1 indicated that perceptually distinct task features other than location did not eliminate backward inhibition. Experiment 2 indicated that when switches across task and location were manipulated independently (i.e., all tasks appeared at all locations), backward inhibition was observed for task switches even when consecutive trials appeared at different spatial locations, ruling out eye movement as the source of the difference. The third experiment indicated that when component tasks involved judgments of spatial location, backward inhibition was observed across unique task-location switches. These results indicate that sequential inhibition is a very flexible mechanism that is sensitive to the amount of interference from previous tasks.
\end{abstract}

The task-switching paradigm, in which participants perform a number of simple tasks in sequence, was developed as a means for investigating cognitive control of thought and action (e.g., Allport, Styles, \& Hsieh, 1994). This paradigm has been very productive, indicating several intentional and stimulus-controlled processes involved in multitask performance. Much of the research in which this paradigm has been used has indicated that control processes are very context sensitive, recruiting different processes for the control of action in different situations.

One control process that appears to be particularly context sensitive is the process associated with suppressing competition from recently performed tasks. The method developed by Mayr and Keele (2000) to observe sequential inhibition involves switches among three tasks and shows that alternating tasks in a three-trial series (e.g., ABA) is both slower and more error prone than switching between three separate tasks (e.g., CBA). The difference between two-switch and alternating-switch sequences is known as backward inhibition (Mayr \& Keele, 2000). ${ }^{1}$

Backward inhibition has been observed with several perceptual and semantic judgment tasks (e.g., Arbuthnott \& Frank, 2000; Koch, Gade, \& Philipp, 2004; Mayr \& Keele, 2000), as well as in several contexts (e.g., Gade \& Koch, 2005; Koch, Philipp, \& Gade, 2006; Mayr \& Kliegl, 2003; Philipp \& Koch, 2006; Schuch \& Koch, 2003). However, one manipulation that eliminates backward inhibition is to present component tasks at different spatial locations (Arbuthnott, 2005; Arbuthnott \& Woodward, 2002). In the experiments in Arbuthnott (2005), switches among three types of digit judgment (magnitude, parity, and prime number status) were precued either by using verbal cues (i.e., less/more, odd/even, and prime/multiple) or by presenting each task at a unique location on a computer screen (e.g., top center for magnitude, lower right for parity, and lower left for prime number judgment). Verbally cued trials, which were presented in a single location on the screen, showed significant backward inhibition, but spatially cued trials showed alternating-switch facilitation (see Figure 1, which presents combined data from these experiments). This pattern is typical for the comparison between two-switch and alternating-switch trials, indicating that there is no inhibition of previous task sets when tasks are presented at unique spatial locations.

The present experiments were designed to test three hypotheses for why spatial cuing eliminates backward inhibition: task set distinctiveness, movement of attention and vision across location, and general location-specific stimulus-response binding. Arbuthnott (2005) speculated that backward inhibition was eliminated with uniquely localized tasks because location decreases competition among the task sets, eliminating the need for sequential inhibition. If this hypothesis is correct, it should be possible to decrease between-task competition by other means. In Experiment 1, we tested this by providing external cues other than spatial location to disambiguate task identification. Specifically, cues and/or targets for each task were presented in distinct colors, providing unique perceptual features that were associated with each task. Distinct color did not reduce backward inhibition, so in Experiment 2, we tested the hypothesis that movement across spatial location uniquely influences inhibition processes. If move-

K.Arbuthnott, katherine.arbuthnott@uregina.ca 
Switch Condition by Cue Type

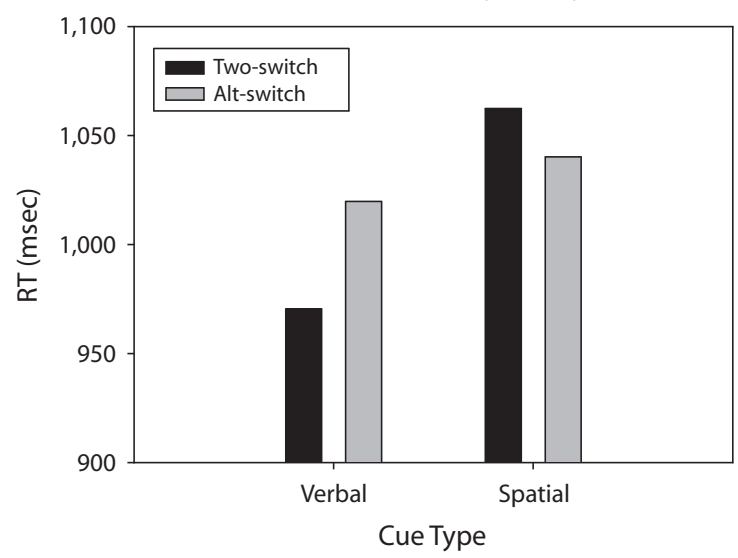

Figure 1. Response times (RTs) for task switches with verbal versus spatial task cues. Alt-switch, alternating-switch condition.

ment of vision or attention between locations eliminates sequential inhibition, this would be evident when shifts of spatial attention are made independently of task switches. In Experiment 2, we examined this possibility by manipulating spatial switches and task switches independently. The results of this experiment showed that switches between tasks, but not locations, resulted in backward inhibition, indicating that shifts of spatial location per se are not the relevant factor in this effect. In Experiment 3, we then examined the generality of backward inhibition elimination with uniquely located tasks by examining switches among three spatial judgment tasks. In contrast to previous results, significant backward inhibition for spatially cued tasks was shown in this situation, indicating that sequential inhibition operates across spatial location when location is relevant to task performance.

\section{EXPERIMENT 1}

As a possible explanation for the effect of spatial cuing on backward inhibition, Arbuthnott (2005) speculated that facilitating task identification by presenting each task in a unique location may reduce the activation of competing categories or responses and, thus, reduce the need for sequential inhibition during response selection. In other words, it was hypothesized that more perceptually discriminable task representations resulted in greater activation of the current task set, relative to previous sets, reducing the need for inhibition to resolve set competition. If this is why spatial cues eliminate backward inhibition, other manipulations that increase the perceptual discriminability of component tasks should show the same effect.

Early work in the task-switching paradigm indicated that switch cost is not observed when a stimulus uniquely specifies which component task to do (Jersild, 1927; Spector \& Biederman, 1976). Spatially defined tasks may partially replicate the situation of unique stimuli, in the same way that digits are interpreted differently depending on their location in a multidigit number (e.g., as seven in the number 47 , but as seventy in the number 78). Thus, it is possible that manipulations such as spatial cuing influence stimulus representations for the different tasks, reducing cross-task stimulus-response conflict. This would reduce the need to suppress competing task sets by more rapidly increasing activation of the relevant task set.

Experiment 1 tested whether a factor other than spatial location - namely, color — can similarly reduce backward inhibition. With spatial cues, both the cue and the target digit appear at distinct locations, so both cues and targets were manipulated in this experiment. Previous evidence has indicated that sequential inhibition occurs after the target stimulus has appeared, rather than during the preparation interval (Hübner, Dreisbach, Haider, \& Kluwe, 2003; Mayr \& Kliegl, 2003), suggesting that it may be the target, rather than the cue, that is relevant to reduced backward inhibition. Furthermore, switching cues without switching tasks increases switch cost (Logan \& Bundesen, 2003) but does not influence backward inhibition (Gade \& Koch, in press; Mayr \& Kliegl, 2003). Given these findings, distinct targets may influence backward inhibition more than do distinct cues, so distinct cues and targets were tested independently. For one group of participants, verbal cues were presented in different colors (red, blue, or green), and the target stimuli were presented in a constant color (black). For a second group, target stimuli for each task were presented in a different color, whereas verbal cues were presented in a constant color. To mimic the situation with spatial cues as much as possible, a third group received both cues and targets presented in distinct colors for each task. Furthermore, the cues in the third group were colored asterisks, requiring the participants to rely on color to determine which task to perform, similar to the situation with spatial cues in Arbuthnott (2005). If task distinctiveness is the relevant factor in the reduction of backward inhibition with spatially cued tasks, these conditions should show reduced backward inhibition.

\section{Method}

Participants. Sixty-four participants (49 women) were recruited from the participant pool operated by the University of Regina psychology department. The participants ranged in age from 18 to 47 years $(M=21.59, S D=5.89)$, reported normal or correctedto-normal vision, and spoke English as their first language. They received course credit in an introductory psychology class in exchange for their participation. The study took approximately $60 \mathrm{~min}$ to complete. The participants were randomly assigned to the colored cue $(n=16)$, colored target $(n=14)$, or colored cue and target $(n=34)$ conditions. Twice as many participants were assigned to the combined condition, because this condition most completely matched spatial cue conditions and, thus, provided the strongest test of the hypothesis. The same tasks and procedures were used as those in the verbal cue conditions of Arbuthnott (2005), except for the addition of colored cues and targets.

Stimuli and Design. Stimulus digits included 2, 3, 4, 6, 7, and 9, which provide equal numbers of stimuli for each bimodal judgment without complete category overlap for the three tasks. A single digit was displayed on each trial. To indicate which judgment to perform, precues were displayed $1,000 \mathrm{msec}$ prior to the appearance of the target digit. For the distinct cue and distinct target conditions, response option cues (i.e., less/more, odd/even, prime/multiple) were used (visual angles, approximately $4^{\circ} \times 0.7^{\circ}$ ). For the distinct cue/ 
target condition, seven colored asterisks were used to precue each trial (visual angles, approximately $3^{\circ} \times 0.7^{\circ}$ ). For the colored cue conditions, the cues were presented in unique colors for each task: red for magnitude judgment, blue for parity judgment, and green for prime number judgment. These colors were chosen to match the covers on the response keys for each task. In the distinct cue condition, the targets were always presented in black. For the colored target condition, the target stimuli were presented in unique colors for each task, using the same task-color assignment as that in the colored cue condition, and all the cues were presented in black. In the colored cue/target condition, both the cues and the targets were presented in the same color-task combinations as in the other conditions.

Six blocks of 72 trials were presented to each participant. The task for each trial was selected randomly, resulting in four possible 3-trial sequences: alternating switches (e.g., BAB), two switches (e.g., CAB), one switch (e.g., AAB), or no switch (e.g., ABB). The design of the study was 3 (distinct feature: cue, target, or cue/target) $\times 4$ (switch condition). The distinct feature factor was tested between participants, and the switch condition factor was tested within participants.

Apparatus and Procedure. The participants were tested individually in a small room with the experimenter present. The stimuli were presented on an IBM-compatible computer connected to a monitor that displayed black characters against a white background. Cues were presented slightly above the vertical center of the screen, and the digit stimulus, about $6 \times 6 \mathrm{~mm}$ in size (visual angle, approximately $0.7^{\circ} \times$ $0.7^{\circ}$ ), was presented immediately below and centered on the cue. For each trial, the cue appeared and remained on screen for $1,000 \mathrm{msec}$ and then was joined by the digit stimulus, which remained on screen until the participant's keypress response. Both the digit and the task were randomly selected, without replacement, from a set of 72 trials representing four repetitions of each cue-target pairing.

Responses were made using all keys on a six-button response box, accurate to $1 \mathrm{msec}$. Unique response options were used for each task to enable discrimination of wrong-task errors (e.g., judging 3 to be odd on a magnitude judgment trial) and decision errors (e.g., judging 3 to be more than 5 on a magnitude judgment trial). Responses were made using the same left and right fingers for each task: Magnitude judgment responses were made with the ring fingers (left for less, right for more), prime judgments were made with the middle fingers (left for prime, right for multiple), and parity judgments were made with the index fingers (left for odd, right for even). The left/right orientation of the responses were chosen to match the order for the response option cues. Each pair of keys (i.e., task assignment) was marked with a different color (red for magnitude judgment, blue for prime judgment, and yellow for parity judgment).

The cue for the next trial appeared $50 \mathrm{msec}$ after the participant's response (i.e., 50-msec response-cue interval [RCI]). A short RCI was chosen to maximize backward inhibition, enabling more sensitive discrimination of the effect of task discriminability. The participants were instructed to make their responses as quickly and accurately as possible and were encouraged to use the precues to prepare for the upcoming trial in order to respond most efficiently. The task-color associations were not explicitly mentioned to the participants but were demonstrated on instruction screens and during practice trials. For trials on which an error was made, the error was recorded and the trial was re-presented immediately, to prevent loss of trials following an error. The response time (RT) data for the second presentation of the error trial were not collected.

In order to familiarize them with the procedure and judgment tasks, the participants were initially given 105 training and practice trials. The order of single-task practice was magnitude, prime, and parity judgments. Following this, 75 mixed task trials were presented. Then the six blocks of experimental trials were presented, with a short break between blocks.

\section{Results}

Trials were coded for switch condition by designating the two three-trial sequences (i.e., two switches [CAB] and alternating switches [BAB]) first. The remaining trials were then designated as no-switch (e.g., ABB or BBB) or one-switch (e.g., AAB) trials. With this procedure, each trial was included in only one switch condition. Due to unconstrained randomization of tasks, several consecutive no-switch trials could occur. On average, each of the switch conditions (one switch, two switches, and alternating switches) occurred on approximately $22 \%$ of the trials, and approximately $32 \%$ of the trials were no-switch trials. ${ }^{2}$ RTs more than $2.5 \mathrm{SD}$ s from each switch condition mean were discarded as outliers (approximately $2.6 \%$ of the trials). Mean RTs and error rates for each condition are presented in Table 1.

Backward inhibition. Backward inhibition is calculated by comparing the performance for alternatingswitch sequences (ABA) with that for two-switch sequences (CBA). Correct RTs were analyzed using a 3 (distinct feature: cue, target, or cue/target) $\times 2$ (switch condition) ANOVA. This analysis indicated only a main effect of switch condition $[F(1,61)=10.01, p=.002]$. Responses were faster in the two-switch condition (means of $977 \mathrm{msec}$ for two switches vs. $1,029 \mathrm{msec}$ for alternating switches). Post hoc $t$ tests indicated that backward inhibition was significant for all three groups $[t(15)=2.16$, $p=.047$ (43 sec), for distinct cues; $t(13)=3.26, p=.006$ $(60 \mathrm{msec})$, for distinct targets; and $t(33)=2.06, p=.048$ (53 msec), for distinct cues/targets].

The parallel analysis of wrong-task errors indicated a similar main effect of switch condition $[F(1,61)=13.43$, $p=.001]$, with more wrong-task errors in the alternatingswitch condition $(3.58 \%$ for two switches vs. $4.56 \%$ for alternating switches). The interaction was also significant $[F(1,61)=3.69, p=.031]$, because significant backward inhibition was observed only in the distinct target condition $[t(13)=2.55, p=.024 ; 2.60 \%$ for distinct targets vs. $0.80 \%$ for distinct cues and $0.40 \%$ for both]. The analysis of decision error rates indicated no significant effects.

Table 1

Mean Response Times (RTs, in Milliseconds) and Error Rates (\%) by Cue Type and Switch Condition in Experiment 1

\begin{tabular}{|c|c|c|c|c|c|c|}
\hline \multirow[b]{2}{*}{ Condition } & \multicolumn{2}{|c|}{ RT } & \multicolumn{2}{|c|}{ WT Error } & \multicolumn{2}{|c|}{ D Error } \\
\hline & $M$ & $S E$ & $M$ & $S E$ & $M$ & $S E$ \\
\hline \multicolumn{7}{|c|}{ Distinct Cues } \\
\hline No switch & 672 & 30.4 & 2.7 & 0.6 & 3.0 & 1.3 \\
\hline One switch & 917 & 14.9 & 6.7 & 2.1 & 5.1 & 1.2 \\
\hline Two switches & 958 & 15.0 & 6.2 & 1.7 & 4.2 & 1.5 \\
\hline Alternating switches & 1,001 & 16.6 & 7.0 & 1.7 & 4.9 & 1.4 \\
\hline \multicolumn{7}{|c|}{ Distinct Targets } \\
\hline No switch & 670 & 32.6 & 1.6 & 0.9 & 5.5 & 0.9 \\
\hline One switch & 946 & 14.7 & 4.0 & 0.5 & 6.7 & 0.7 \\
\hline Two switches & 992 & 13.1 & 4.3 & 0.4 & 6.4 & 1.0 \\
\hline Alternating switches & 1,052 & 15.6 & 6.9 & 1.1 & 5.9 & 0.7 \\
\hline \multicolumn{7}{|c|}{ Distinct Cues/Targets } \\
\hline No switch & 636 & 30.1 & 0.7 & 0.4 & 5.2 & 0.4 \\
\hline One switch & 991 & 12.3 & 1.7 & 0.5 & 6.0 & 0.6 \\
\hline Two switches & 1,013 & 14.6 & 2.0 & 0.5 & 5.7 & 0.6 \\
\hline Alternating switches & 1,062 & 18.1 & 2.4 & 0.4 & 6.5 & 0.5 \\
\hline
\end{tabular}

Note-WT, wrong-task; D, decision. 
Switch cost. To examine the influence of distinctiveness on switch cost, no-switch and one-switch trials were compared using 3 (distinct feature) $\times 2$ (switch condition) ANOVAs. The analysis of RTs indicated a main effect of switch condition $[F(1,61)=116.34, p<.001]$, showing a typical switch cost effect ( $645 \mathrm{msec}$ for no switch vs. $946 \mathrm{msec}$ for one switch). Switch cost was significant for all three groups $[t(15)=5.96, p<.001(245 \mathrm{msec})$, for distinct cues; $t(13)=6.06, p<.001(276 \mathrm{msec})$, for distinct targets; and $t(33)=9.01, p<.001(339 \mathrm{msec})$, for distinct cues/targets].

The 3 (distinct feature) $\times 2$ (switch condition) analyses of error rates indicated only main effects of switch condition for both wrong-task errors $[F(1,61)=15.27$, $p<.001]$ and decision errors $[F(1,61)=6.89, p=.011]$, with more errors in the one-switch condition. Nominally, switch cost was greater with wrong-task errors $(2.05 \%$ cost) than with decision errors $(1.18 \%$ cost $)$. Neither the main effect of feature nor the interaction was significant in either analysis.

\section{Discussion}

Experiment 1 was conducted to determine whether non-spatially-distinct cues and/or targets would reduce the magnitude of backward inhibition, similar to the effect of spatial cues. The results indicated that this was not the case. A pilot study comparing distinct and uniform cues showed improved overall performance with distinct cues (means of 1,023 msec for distinct cues vs. 1,236 msec for uniform cues), indicating that distinct features did influence the participants' performance. However, as with the results of Experiment 1, equivalent backward inhibition was observed for both distinct and uniform cues. Thus, task distinctiveness per se does not explain the effect of spatial cues on backward inhibition.

The absence of group differences, especially for those who received both distinct cues and targets, is somewhat surprising. However, the cues for the distinct cue/target group were abstract, as compared with the response option cues for the other two groups. The greater difficulty retrieving task set with the abstract cues (Mayr \& Kliegl, 2000) may have overshadowed any priming advantage associated with consistent cue and target colors.

Given that nonspatial task distinctiveness did not eliminate backward inhibition, it appears that the spatial dimension is key to this effect. One straightforward possibility is that shifts of location themselves eliminate the operation of sequential inhibition.

\section{EXPERIMENT 2}

The purpose of Experiment 2 was to test the hypothesis that shifting vision and attention across location eliminated sequential inhibition of competing tasks. In this experiment, switches across task and location were manipulated independently. Three different locations were used, as in earlier studies (Arbuthnott, 2005; Arbuthnott \& Woodward, 2002), but in this experiment, location was not associated with task, since all three tasks were presented at each location.

\section{Method}

Participants. Thirty-eight participants ( 28 women) were recruited from the participant pool operated by the University of Regina psychology department. Participants ranged in age from 18 to 48 years $(M=21.16, S D=6.57)$ and received course credit in introductory psychology classes in exchange for their participation. The participants reported normal or corrected-to-normal vision and spoke English as their first language. The study took approximately $45 \mathrm{~min}$ to complete.

Stimuli and Design. The same tasks and stimuli as those in Experiment 1 were used. Trials were presented at one of three locations on the computer screen (top center, bottom left, and bottom right), and all three tasks were presented at each location across trials. The three screen locations defined a $10-\mathrm{cm}$ equilateral triangle with the apex of the triangle at the top of the screen (approximately $11^{\circ}$ of visual angle per side of the virtual triangle). Two switch types were represented in this experiment: task switches and location switches. For both, the four conditions were the same as those in Experiment 1 (no switch, one switch, two switches, and alternating switches). For task switches, switch referred to changes of the judgment task across trials. Verbal response option precues, presented at the location of the next trial, were used to indicate which task to perform. For location switches, switch referred to changes of location across trials. For instance, in the location no-switch condition, consecutive trials were presented at the same location on the screen, regardless of the task assignment for the consecutive trials. Similarly, in the task noswitch condition, consecutive trials involved the same task, regardless of whether location switched or not.

Task, target digit, and location were randomly selected, without replacement, from a set of 108 trials representing 2 trials of each digit/location/task combination. Four experimental blocks of 108 trials were presented to each participant. The design of the study was 4 (location shift) $\times 4$ (task switch), and all factors were manipulated within participants.

Apparatus and Procedure. The same apparatus and procedures were used as those in the previous experiment (and in Arbuthnott, 2005), with two exceptions: (1) Cues and stimuli were presented at three different locations on the screen (as in the spacial cue condition in Arbuthnott, 2005), and (2) only two response keys were used, to reduce task complexity for the participants. For each trial, the digit stimulus was presented immediately below and centered on the cue. Responses were made using two keys on the six-button response box, using the same left/right orientation as that for the response option cues: left for less, odd, and prime judgments and right for more, even, and multiple judgments.

To familiarize them with the procedure, the participants initially received 105 training and practice trials. For each task, 10 consecutive trials were presented, with location on the screen varying randomly. The order of single-task practice was magnitude, prime, and parity judgment. Following this, 75 mixed task and location trials were presented. Following the practice block, four blocks of experimental trials were presented, with a short break between blocks.

\section{Results}

Two participants were unable to complete the task, and their data were discarded from the analysis. Trials were coded for location switch in the same manner as for task switches, designating the two 3-trial sequences first. RTs more than $2.5 S D$ s from each location switch $\times$ task switch condition mean were discarded as outliers (approximately $2.4 \%$ of the trials). Mean correct RTs and error rates for each condition are presented in Table 2.

Location and task switches were tested orthogonally, so 4 (task switch) $\times 4$ (location switch) ANOVAs of RTs and error rates were conducted. The RT analysis indicated significance for both main effects $[F(3,105)=51.99, p<.001$, 
Table 2

Mean Response Times (R'Is, in Milliseconds) and Error Rates (\%) by Location Switch and Task Switch Conditions in Experiment 2

\begin{tabular}{|c|c|c|c|c|c|c|c|c|c|c|c|c|c|c|c|c|}
\hline \multirow[b]{4}{*}{ Task Switch } & \multicolumn{16}{|c|}{ Location Switch } \\
\hline & \multicolumn{4}{|c|}{ No Switch } & \multicolumn{4}{|c|}{ One Switch } & \multicolumn{4}{|c|}{ Two Switches } & \multicolumn{4}{|c|}{ Alternating Switches } \\
\hline & \multicolumn{2}{|c|}{ RT } & \multicolumn{2}{|c|}{ Error } & \multicolumn{2}{|c|}{ RT } & \multicolumn{2}{|c|}{ Error } & \multicolumn{2}{|c|}{ RT } & \multicolumn{2}{|c|}{ Error } & \multicolumn{2}{|c|}{ RT } & \multicolumn{2}{|c|}{ Error } \\
\hline & $M$ & $S E$ & $M$ & $S E$ & $M$ & $S E$ & $M$ & $S E$ & $M$ & $S E$ & $M$ & $S E$ & $M$ & $S E$ & $M$ & $S E$ \\
\hline No switch & 653 & 24 & 5.69 & 0.8 & 700 & 31 & 6.93 & 0.9 & 670 & 25 & 6.19 & 1.0 & 689 & 25 & 6.66 & 1.0 \\
\hline One switch & 788 & 36 & 8.38 & 1.1 & 834 & 42 & 9.17 & 1.1 & 828 & 38 & 8.90 & 1.5 & 798 & 38 & 8.38 & 1.4 \\
\hline Two switches & 817 & 39 & 7.65 & 1.1 & 856 & 46 & 10.51 & 1.7 & 880 & 43 & 9.10 & 1.8 & 805 & 39 & 9.20 & 1.3 \\
\hline Alternating switches & 795 & 34 & 7.84 & 1.0 & 913 & 49 & 9.71 & 1.3 & 894 & 51 & 10.45 & 1.1 & 859 & 39 & 10.49 & 1.4 \\
\hline
\end{tabular}

for task switch; $F(3,105)=10.33, p<.001$, for location switch; $F(9,315)=2.03, p=.035$, for the interaction].

The main effect of task switch indicated that no-switch RT $(678 \mathrm{msec})$ was shorter than those for all the switch conditions $(812,839$, and $866 \mathrm{msec}$ for one switch, two switches, and alternating switches, respectively; Tukey's honestly significant difference $[\mathrm{HSD}]=86.47$ ). In contrast, the main effect of location switches was less straightforward. Responses on location no-switch trials were fastest $(763 \mathrm{msec})$ but did not significantly differ from those for location alternation $(788 \mathrm{msec})$, and responses in both of these conditions were faster than those in the one-switch $(826 \mathrm{msec})$ and two-switch $(818 \mathrm{msec})$ conditions $(\mathrm{HSD}=$ 67.05). Thus, alternating location nominally facilitated performance, relative to the other location switch conditions.

The significant interaction between task switches and location switches indicted a complex pattern. For all location switch conditions, responses for task repetitions (noswitch trials) were faster than those for all the task switch conditions (HSD $=59.08)$. Within the task switch conditions, significant differences showed no obvious pattern. For instance, responses in the task alternating-switch/ location one-switch and two-switch conditions were slower than those in the task alternating-switch/location no-switch condition, but they were also slower than those in the task one-switch/location no-switch and task twoswitch/location alternating-switch conditions. For all the location switch conditions except the no-switch condition, task alternation resulted in the longest RTs, but long RTs were also observed in the task two-switch/location twoswitch condition.

Since the responses were multivalent, only combined error rates could be examined, and the analysis of error rates indicated only a main effect of task switch $[F(3,105)=10.12, p<.001]$. Error rates were lower in the task no-switch condition $(6.37 \%)$ than in all the task switch conditions $(8.71 \%, 9.12 \%$, and $9.62 \%$ for the oneswitch, two-switch, and alternating-switch conditions, respectively; HSD = 3.39).

The primary purpose of this experiment was to determine whether location switches themselves caused the elimination of backward inhibition. The main effect of location switches, with facilitation in the alternatinglocation condition, relative to location two-switch trials, suggests that this was not the case. However, given the complex interaction between task switches and location switches, comparisons of backward inhibition and switch cost for the two switch types, collapsed across the other factor, were also conducted in order to more clearly elucidate the effect of switch type on backward inhibition.

Backward inhibition. To compare the two switch types for alternating-switch and two-switch conditions, RTs and error rates ${ }^{3}$ were collapsed across all the other conditions for each switch type. A 2 (switch type: task vs. location) $\times 2$ (switch condition: two switches vs. alternating switches) ANOVA indicated a main effect of switch type $[F(1,35)=45.47, p<.001]$ and an interaction between the two factors $[F(1,35)=10.08, p=.003]$. The main effect arose because responses for location switches were faster $(803 \mathrm{msec})$ than those for task switches $(852 \mathrm{msec})$. The interaction arose because there was a mean $26-\mathrm{msec}$ cost for task alternation but a mean 30 -msec facilitation for location alternation. These differences were significant for both task switches $[t(35)=2.23, p=.033]$ and location switches $[t(35)=2.24, p=.032]$.

The analysis of error rates indicated no significant effects (all $F_{\mathbf{s}}<1$ ). The mean error rates for the two-switch and alternating-switch conditions were $8.57 \%$ and $8.67 \%$, respectively.

Switch cost. To examine how location switches and task switches influenced switch cost, RTs for no-switch and one-switch trials (computed as for two-switch and alternating-switch trials) were compared using a 2 (switch type) $\times 2$ (switch condition) ANOVA. Both main effects and the interaction were significant. The effect of switch condition $[F(1,35)=78.02, p<.001]$ indicated the typical effect of slowed performance after a switch (mean of $822 \mathrm{msec}$ ), as compared with repeated task or location (mean of $721 \mathrm{msec})$. The effect of switch type $[F(1,35)=$ 54.93, $p<.001]$ indicated that responses on task-coded trials were faster $(745 \mathrm{msec})$ than those on location-coded trials $(798 \mathrm{msec}$ ). The main effect of switch type, however, was largely attributable to longer RTs for location repetitions $(763 \mathrm{msec})$ than for task repetitions $(678 \mathrm{msec})$, as indicated by the significant interaction $[F(1,35)=9.33$, $p=.004]$. In other words, it was more costly to switch tasks (134 msec) than to switch locations (69 msec). Unlike the situation for backward inhibition, however, the direction of the effects for both task and location switches was the same: benefit for repetition and cost for switching.

The parallel analysis of error rates indicated main effects of both switch condition $[F(1,35)=21.61, p<.001]$ and switch type $[F(1,35)=7.65, p=.009]$. Errors were more frequent for switch trials (means of $6.88 \%$ for no- 
switch and $8.68 \%$ for one-switch trials), and for locationcoded trials $(8.23 \%)$ than for task-coded trials $(7.32 \%)$. The latter undoubtedly occurred because location noswitch trials most often involved a task switch $(64 \%$ of trials), whereas task no-switch trials involved less costly shifts of location.

\section{Discussion}

The purpose of Experiment 2 was to examine whether moving one's eyes or attention between locations eliminates backward inhibition. In contrast to this prediction, alternating location across three-trial sequences facilitated, rather than impaired, performance. Alternating tasks, however, generally resulted in backward inhibition. This suggests that, across three-trial sequences, task sets are inhibited when a new task is required but that locations are not. The observation of backward inhibition for task switches, even though component tasks were presented at different locations, indicates that sequential inhibition operates between tasks even when they do not share a single spatial location, as long as the tasks are not uniquely associated with location.

The absence of backward inhibition in the location no-switch condition (see Table 2) seems unusual because backward inhibition has previously been observed when tasks are presented at a consistent location. However, the situation in this experiment differed from methods with a consistent location. Specifically, in this experiment, location no-switch trials usually involved a different location on trial $n-2$ (i.e., locations ABB), which would be the alternating-task trial. Thus, a return to a task that was previously performed at a different location was apparently easier than retrieval of a task that was not performed on the previous two locations (i.e., task two-switch/location noswitch trials). Other than this difference, however, the reason for the 22-msec facilitation for task alternation in the location no-switch condition is not clear. The only conditions in this experiment that matched the local conditions in previous studies with spatial cues were the two threetrial sequences with parallel task and location switches (i.e., task and location two-switch and alternating-switch conditions). In these conditions, either tasks appeared in consistent alternating locations, or three different tasks appeared in three different locations. In previous studies, these conditions showed benefit for alternating switches, as compared with two switches, and this was also nominally observed in this study, at least for RTs $(880 \mathrm{msec}$ for the two-switch combination vs. $859 \mathrm{msec}$ for the alternating-switch combination).

The results of this experiment suggest that it is not shifts of location per se but, rather, unique task-location associations that cause the elimination of backward inhibition. Recently, Mayr and Bryck (2007) observed that presenting tasks at unique locations reduced switch costs, at least in error rates. Furthermore, response repetition costs, typically observed for task switch trials, were not observed for the localized tasks. Mayr and Bryck used only two tasks, so backward inhibition could not be examined, but they attributed switch cost reduction for localized tasks to low-level stimulus-response binding that was distinct for each location, thus reducing between-task response competition. Mayr and Bryck argued that localized objects may have a privileged role in constraining action-relevant representations. The disconfirmation of the alternative distinctiveness and spatial movement hypotheses provide indirect support for this location-specific stimulus-response binding mechanism. However, before fully accepting this as the source of the elimination of backward inhibition with spatial cues, the generality of this influence must be tested.

In all the previous experiments showing the elimination of backward inhibition with spatial task cues, the tasks involved categorical judgments. In this context, backward inhibition was not observed for categorical task sets that were uniquely associated with location, consistent with the view of Mayr and Bryck (2007). However, it remains to be seen whether this influence of location on backward inhibition is observed in all contexts, especially with switches between spatial judgment tasks.

\section{EXPERIMENT 3}

The purpose of Experiment 3 was to examine whether backward inhibition would be observed across spatial location with spatially distinct tasks that involve location judgment. To test this, participants performed three location judgment tasks (up/down, left/right, and near/far), with stimuli presented either centrally for all tasks (verbally cued) or at unique locations for each task (spatially cued).

\section{Method}

Participants. Thirty-nine participants (30 women) were recruited from the participant pool operated by the University of Regina psychology department. The participants ranged in age from 18 to 56 years $(M=22.26, S D=6.76)$, reported normal or correctedto-normal vision, and spoke English as their first language. They received course credit in an introductory psychology class in exchange for their participation. The study took approximately $60 \mathrm{~min}$ to complete.

Stimuli and Design. The tasks used in this experiment involved judgment of the location of an asterisk $\left({ }^{*}\right)$ relative to a point marked with intersecting lines $(+)$. Three different location judgments were used: up/down, left/right, and near/far. To indicate which judgment was required, the participants received either a verbal precue (up/ down, left/right, or near/far) or a spatial cue (up/down judgment at the top center of the screen, left/right judgment at the bottom left, and near/far judgment at the bottom right), as in the verbal- and spatial-cuing conditions in Arbuthnott (2005). In the spatial cue condition, the marker $(+)$ appeared at one of the screen locations prior to target presentation to allow preparation of the appropriate task.

The target stimulus appeared at one of eight different locations relative to the marker, representing a factorial combination of all the possible judgments. For instance, the asterisk could appear above, to the left, and far from the location marker, for each of the three tasks. Relative to the marker, the targets were equally presented above or below, to the left or right, and close to or far from the marker.

For the verbally cued condition, the marker $(+)$ was located $26 \mathrm{~mm}$ beneath the verbal cue (visual angle, $0.2^{\circ}$ ), and the target stimulus appeared in relation to the marker. Near stimuli were presented $7 \mathrm{~mm}$ (visual angle, $0.06^{\circ}$ ) from the center point of the marker in both directions (i.e., above/below and left/right), and far stimuli were presented $13 \mathrm{~mm}$ (visual angle, $0.11^{\circ}$ ) from the central marker in both directions. The eight target locations thus defined a virtual $\mathrm{X}$, with the marker at the cross point. For the spatial cue condition, 


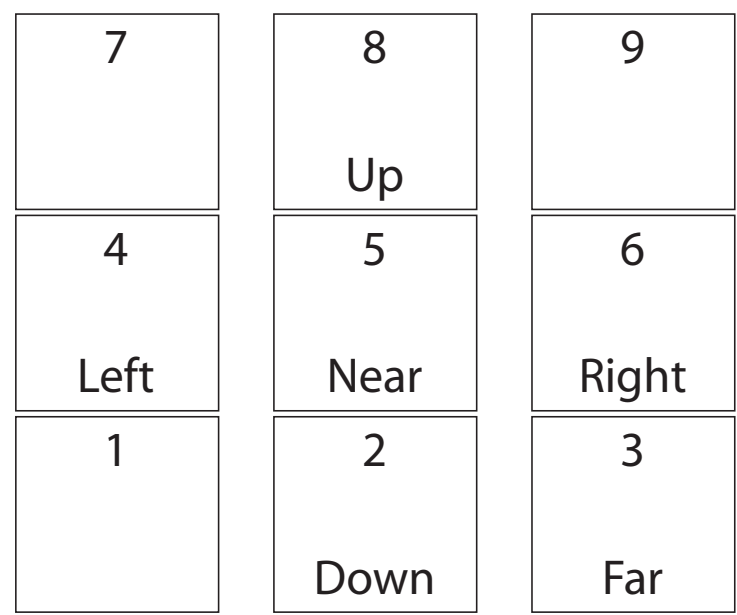

Figure 2. Response key arrangement, Experiment 3.

the marker appeared in one of the three locations defined by a virtual 12-cm equilateral triangle centered on the computer screen (approximately $11^{\circ}$ of visual angle per side of the virtual triangle), with the apex of the triangle at the top of the screen. The target stimuli were presented at the same distances and locations, relative to the marker, as in the verbal cue condition.

Cue type was manipulated within participants, with order counterbalanced across participants. Half of the participants first received the verbally cued trials, in which the marker was located at screen center. The other participants first received the spatially cued trials, in which the marker was located at one of three screen locations. The design was a 2 (cue type) $\times 4$ (switch condition) factorial, with both factors manipulated within participants.

Apparatus and Procedure. The apparatus and testing procedure were the same as those in the previous experiments, including the 50-msec RCI and 1,000-msec cue-task interval. For each trial, the cue (either the verbal cue plus the marker or the marker alone) appeared and remained on screen for 1,000 msec and then was joined by the target asterisk, which remained on screen until the participant's keypress response. Responses were made using one of six keys on the number pad of a standard keyboard. Up/down responses were made using the " 8 " or " 2 " keys, left/right responses were made with the " 4 " and " 6 " keys, and near/far responses were made using the " 5 " and " 3 " keys (see Figure 2).

Eight blocks of 72 trials were presented to each participant, four blocks with each cue type. For each trial, the task and stimulus location, relative to the marker, was randomly selected, without replacement, from a set of 72 task/location combinations. Within each block, the target asterisk appeared in each possible location three times for each task.
To familiarize them with the judgment tasks and the precues, the participants were given 105 training and practice trials immediately prior to each of the four experimental blocks with a given cue type. For each practice, 10 consecutive trials of each task were presented prior to the experimental blocks of each cue type (in the order up/ down, left/right, and near/far judgments), followed by 75 mixed task trials. For the spatial cue condition, verbal cues were presented above the marker for the first 15 mixed task trials, to facilitate the participants' learning the location-task associations. Practice trials of a given cue type were followed by the four experimental blocks of that cue type.

\section{Results}

The data from 2 participants were excluded from analysis because their mean error rate in one of the cue type conditions exceeded $20 \%$. Trials were coded for switch condition as in the previous experiments, designating the two three-trial sequences first. RTs more than $2.5 \mathrm{SDs}$ from each cue type $\times$ switch condition mean were discarded as outliers (approximately $2.9 \%$ of the trials). Mean correct RTs and error rates for each condition are presented in Table 3.

Backward inhibition. To examine the effect of switching between spatial judgment tasks on backward inhibition, correct RTs were analyzed using a 2 (cue type: verbal or spatial) $\times 2$ (switch condition: two switches or alternating switches) ANOVA. This analysis indicated only a main effect of switch condition $[F(1,36)=7.62, p=.009]$. Responses were faster in the two-switch condition (means of $759 \mathrm{msec}$ for two-switch vs. $785 \mathrm{msec}$ for alternatingswitch trials). Backward inhibition was observed for both cue type conditions (30 msec for verbal cues and $21 \mathrm{msec}$ for spatial cues), although post hoc $t$ tests indicated that the cost was statistically significant only for the verbal cue condition $[t(36)=2.18, p=.036$, for verbal cues; $t(36)=$ $1.63, p=.112$, for spatial cues]. Unlike in the previous experiments, however, backward inhibition, rather than alternating-switch facilitation, was observed when the tasks were uniquely associated with spatial locations.

The parallel $2 \times 2$ ANOVA of wrong-task errors indicated main effects of cue type $[F(1,36)=5.37, p=.026]$ and switch condition $[F(1,36)=10.30, p=.003]$. More wrong-task errors were made in the spatial cue condition (3.19\% for spatial cue vs. $2.18 \%$ for verbal cue) and in the alternating-switch condition (3.21\% for alternatingswitch vs. $2.15 \%$ for two-switch conditions). The interaction was also significant $[F(1,36)=5.57, p=.024]$, because backward inhibition was observed only in the

Table 3

Mean Response Times (RT's, in Milliseconds) and Error Rates (\%) by Cue Type and Switch Condition in Experiment 3

\begin{tabular}{|c|c|c|c|c|c|c|c|c|c|c|c|c|}
\hline \multirow[b]{3}{*}{ Condition } & \multicolumn{6}{|c|}{ Verbal Cues } & \multicolumn{6}{|c|}{ Spatial Cues } \\
\hline & \multicolumn{2}{|c|}{ RT } & \multicolumn{2}{|c|}{ WT Error } & \multicolumn{2}{|c|}{ D Error } & \multicolumn{2}{|c|}{ RT } & \multicolumn{2}{|c|}{ WT Error } & \multicolumn{2}{|c|}{ D Error } \\
\hline & $M$ & $S E$ & $M$ & $S E$ & $M$ & $S E$ & $M$ & $S E$ & $M$ & $S E$ & $M$ & $S E$ \\
\hline No switch & 623 & 13.4 & 1.4 & 0.2 & 1.6 & 0.2 & 601 & 16.9 & 8.2 & 0.3 & 5.0 & 0.3 \\
\hline One switch & 683 & 14.8 & 1.8 & 0.3 & 1.0 & 0.2 & 768 & 15.7 & 2.3 & 0.3 & 0.6 & 0.2 \\
\hline Two switches & 728 & 13.4 & 2.1 & 0.3 & 1.4 & 0.2 & 791 & 19.5 & 2.2 & 0.3 & 0.9 & 0.1 \\
\hline Alternating switches & 758 & 20.5 & 2.3 & 0.3 & 1.8 & 0.3 & 811 & 19.3 & 4.1 & 0.5 & 0.9 & 0.2 \\
\hline
\end{tabular}

Note-WT, wrong-task; D, decision. 
spatial cue condition $[t(36)=3.45, p=.001 ; 1.90 \%$ for spatially cued vs. $0.22 \%$ for verbally cued switches]. Thus, although backward inhibition did not reach significance for RTs in the spatial cue condition, it was significant for wrong-task errors.

The analysis of decision error rates also indicated a significant main effect of cue type $[F(1,36)=11.52, p=.002]$, but in the opposite direction than that for wrong-task errors. More decision errors were made with verbally cued tasks ( $1.61 \%$ for verbal cues vs. $0.88 \%$ for spatial cues). There were no effects of switch condition for decision errors.

Switch cost. To examine the influence of switches among spatial judgment tasks on switch cost, no-switch and one-switch trials were compared using 2 (cue type) $\times$ 2 (switch condition) ANOVAs. The analysis of RTs indicated a main effect of switch condition $[F(1,36)=46.97$, $p<.001]$, showing a typical switch cost effect (612 msec for no switches vs. $726 \mathrm{msec}$ for one switch). Switch cost was significant for both cue types $[t(36)=4.55, p<$ $.001(60 \mathrm{msec})$, for verbal cues; $t(36)=7.30, p<.001$ (168 $\mathrm{msec})$, for spatial cues], and the significant interaction of cue type and switch condition $[F(1,36)=38.42$, $p<.001]$ reflected the larger switch cost for spatially cued trials.

The $2 \times 2$ analyses of error rates indicated main effects of cue type and switch condition for both types of errors. The effect of cue type was similar for both error types, with more errors for spatially cued trials for both wrong-task errors $[F(1,36)=125.01, p<.001 ; 5.41 \%$ for spatial cues vs. $1.48 \%$ for verbal cues] and decision errors $[F(1,36)=51.40, p<.001 ; 2.80 \%$ for spatial cues vs. $1.30 \%$ for verbal cues]. The significant effects of switch condition were modified by interactions between cue type and switch condition for both types of errors $[F(1,36)=$ $179.86, p<.001$, for wrong-task errors; $F(1,36)=59.11$, $p<.001$, for decision errors]. These interactions arose because, for spatially-cued trials, error rates were greater in the no-switch condition $(8.23 \%$ wrong-task errors and $4.97 \%$ decision errors) than for one-switch trials $(2.30 \%$ wrong-task errors and $0.62 \%$ decision errors). Thus, for these trials, there was a switch benefit in accuracy. For verbally cued trials, conversely, no significant differences were observed between no-switch (1.42\% wrong-task and $1.56 \%$ decision errors) and one-switch (1.83\% wrong-task and $1.04 \%$ decision errors) conditions.

To further examine the unexpectedly high error rates on spatially cued no-switch trials, two additional analyses were conducted, both limited to no-switch trials. One possible explanation for this pattern of errors is that the spatial cues themselves created congruence effects, especially for the up/down and left/right tasks. Responses were all congruent, relative to the marker $(+)$, but when tasks were cued by location on the screen, responses consistent with the task location (i.e., up for the up/down task and left for the left/right task) may have added additional interference. For the near/far task, far was coded as congruent for the purpose of this analysis. To test this possibility, 2 (cue type) $\times 2$ (congruence) $\times 3$ (task) ANOVAs were conducted for each error type. If task location interacted with task congruence, this analysis would show a three-way interaction, with congruence on the up/down and left/right tasks having a greater effect in the spatially cued condition. These analyses, however, indicated no significant effects involving congruence $(F$ s $<1$ for the three-way interactions). Thus, this does not appear to be the reason for this error pattern. Consistent with this conclusion, larger error rates for spatially cued no-switch trials were observed in two of the three tasks, but not in the two tasks that would be most influenced by congruence with the task cue. Specifically, up/down (10.51\%) and near/far (12.84\%) judgments showed large error rates, but the left/right judgment $(1.00 \%)$ did not. Thus response congruence with the screen location did not apparently influence error rates.

Another possibility is that no-switch trials that required changing response impaired performance (e.g., Rogers $\&$ Monsell, 1995). To test this possibility, 2 (cue type) $\times$ 2 (response type: repetition vs. switch) ANOVAs of noswitch trials were conducted. These analyses indicated significant interactions for both error types $[F(1,36)=$ $220.54, p<.001$, for wrong-task errors; $F(1,36)=9.69$, $p=.004$, for decision errors]. Errors rates were much higher for spatially cued trials when the participants were required to switch their response (wrong-task error means, $1.17 \%$ vs. $12.84 \%$, and decision error means, $3.79 \%$ vs. $5.94 \%$, for response repetition vs. switch, respectively). Verbally cued trials, in contrast, showed much smaller effects of response type (wrong-task error means, $0.61 \%$ vs. $2.18 \%$, and decision error means, $1.87 \%$ vs. $1.19 \%$, for response repetition vs. switch, respectively). The very large error rate for spatially cued no-switch trials, then, is attributable to the trials that involved response switching.

\section{Discussion}

The purpose of Experiment 3 was to determine whether backward inhibition differed between verbally and spatially cued trials when the component tasks involved spatial, rather than categorical, judgments. In contrast to the previous experiments, cue type did not influence backward inhibition in this context. Spatially cued trials showed backward inhibition, nominally in RTs and significantly in wrong-task error rates. This is the first observation of backward inhibition for switches among spatially isolated tasks and suggests that backward inhibition is sensitive to task characteristics, suppressing localized task sets when location is relevant to task completion.

One surprising feature of these results was the increase in both types of errors for task repetition trials in the spatial cue condition. Post hoc analyses suggest that it was the requirement to switch responses on spatially cued no-switch trials that accounts for this finding. In particular, when the task did not switch but the response did, the participants chose the wrong task, rather than making the other choice within the same task. This finding is similar to the observation that response repetition impairs performance on task switch trials (Rogers \& Monsell, 1995), perhaps due to participants' expectation of concurrent changes in task and response, although this effect seems to have been exaggerated for spatially cued trials. 


\section{GENERAL DISCUSSION}

The purpose of this series of experiments was to test three different hypotheses about why spatial cues eliminate backward inhibition: task set distinctiveness, spatial movement, and the unique task-location associations (specifically, their generality). The results of Experiments 1 and 2 disconfirmed the straightforward distinctiveness and location shift hypotheses, constraining possible sources of this effect. Providing unique color identifiers of tasks did not reduce backward inhibition, and location shifting did not eliminate sequential inhibition for task switches. Experiment 3, however, indicated that backward inhibition is observed for unique task-location combinations when the component tasks involve spatial judgment. Thus, backward inhibition is observed across spatial locations when location is relevant to performance of the task itself. This is the first observation of backward inhibition when tasks are uniquely located and suggests that the mechanism underlying backward inhibition displays considerable flexibility. As was suggested by Mayr and Bryck (2007), location can constrain action-relevant representations, thus eliminating backward inhibition, for switches among category judgment tasks. However, when the representation of spatial location is necessary for task completion, sequential inhibition is observed for uniquely localized tasks.

What have these results contributed to the knowledge of backward inhibition mechanisms? To answer this question, it is necessary to briefly review what was previously known about this effect. First, the magnitude of backward inhibition is sensitive to some types of explicit knowledge. Including task repetitions in a task-switching sequence decreases backward inhibition (Philipp \& Koch, 2006), as does presenting alternating tasks across chunk boundaries (Schneider, 2007) or alerting participants to alternatingtask sequences (Koch et al., 2006). However, backward inhibition cannot be deliberately evoked, since awareness of an upcoming switch does not elicit backward inhibition unless the identity of the upcoming task is known (Hübner et al., 2003). So, although backward inhibition cannot be deliberately used, its magnitude can be modified by explicit knowledge about the advantage of suppressing competing task representations.

Unlike the case for explicit knowledge, both the magnitude and the presence of backward inhibition are sensitive to the magnitude of competitor activation. For instance, longer delays between switch trials reduce backward inhibition (Gade \& Koch, 2005; Mayr, 2002; Mayr \& Keele, 2000) because a longer intertrial interval (ITI) allows activation of the previous task set to decay more than with shorter ITIs. Gade and Koch (2005) determined that this reduction results from reduced suppression of the alternating task set by manipulating RCI independently for task switches on trials $n-1$ and $n$. Backward inhibition was reduced with a longer RCI between trials $n-2$ and $n-1$ but was not influenced by the delay between trials $n-1$ and $n$. This pattern of results indicates that it is the residual activation of the $n-2$ task that influences backward inhibition magnitude, with greater competition leading to increased inhibition.
Similarly, in switches between tasks of unequal difficulty, greater backward inhibition is observed for switches from an easier to a more difficult task (Arbuthnott, in press; see also Philipp, Gade, \& Koch, 2007). In switches among digit judgments of magnitude, parity, and prime number status, magnitude judgment is easier (e.g., RT of $663 \mathrm{msec} ; 2.48 \%$ errors) than judgment of prime number status (e.g., RT of $881 \mathrm{msec} ; 4.31 \%$ errors). Thus, in magnitude-prime-magnitude task switches, magnitude judgment would interfere with prime judgment on trial $n-1$, resulting in greater backward inhibition $(177 \mathrm{msec})$ than for the reverse task sequence ( $41 \mathrm{msec}$ facilitation for prime-magnitude-prime switches).

The present results contribute to understanding the circumstances under which competitor activation influences backward inhibition. Specifically, the results of Experiment 1 indicate that between-task competition is not eliminated by perceptually distinctive task stimuli. The results of Experiment 2 further indicate that competing tasks require sequential inhibition even when they occur at multiple locations, as long as there is not a reliable tasklocation association. By default, the disconfirmation of these alternative hypotheses supports the contention that distinct task-location association eliminates competitor activation and, thus, sequential inhibition of task sets. However, the results of Experiment 3 indicate that even this mechanism does not operate in all situations. Specifically, when spatial location is involved in stimulusresponse binding, sequential inhibition of tasks presented at other locations is necessary.

These observations indicate that the backward inhibition mechanism is extremely flexible, responding selectively to aspects of a task context that cause interference. It is likely that this flexibility arises from a low-level mechanism, such as lateral inhibition of category response rules (Arbuthnott, 2005; Gade \& Koch, 2005; Schuch \& Koch, 2003). This inhibition combines with activation processes to influence performance, which affects whether inhibition or activation dominates in different contexts (Arbuthnott, in press; Gade \& Koch, in press).

The results of Experiment 3 also suggest that the relationship between cue processing and task processing may influence backward inhibition. When component tasks involve categorical judgment, verbally cued trials show backward inhibition, whereas spatially cued trials do not. However, when component tasks involve spatial judgment, spatially cued trials show backward inhibition. Thus, it is possible that cue processing increases interference when there is a match with task processing. However, the finding that both verbal and spatial cue conditions showed backward inhibition for spatial judgment tasks indicates that the match between cue and target processing cannot entirely account for the backward inhibition pattern across different cue types. Specifically, distinct location can eliminate the need for sequential inhibition, but distinct verbal cues cannot, so backward inhibition is observed for verbally cued switches between both category judgment and spatial judgment tasks.

The pattern of results in the present experiments also highlights the uniqueness of location in human cogni- 
tion. Switch cost and backward inhibition are observed, presumably, because holding a single goal in working memory at one time is adaptive (Mayr \& Kliegl, 2000) and it takes time and, often, inhibition to change from one goal to another. However, several important human goals require us to distribute attention across several spatial locations (e.g., search tasks, hunting, attending to children at play, driving), perhaps providing an adaptive advantage to excluding location from suppression of features associated with different goals. If this is the case, sequential inhibition mechanisms may have evolved to benefit sequential performance of category tasks (reducing interference from similar goals), while preserving attention distributed across spatial locations. This suggests that attention to multiple spatial locations may be the normative situation, resulting in location-based inhibition only when attentional resources are severely constrained or when location processing itself is necessary for goal attainment.

\section{AUTHOR NOTE}

This work was supported by the Natural Sciences and Engineering Research Council of Canada. Thanks to Aaron Brown for help with testing participants, to Chris Oriet, Katherine Robinson, and Tom Phenix for fruitful discussions, and to Miriam Gade and Michael Masson for helpful comments on an earlier version of the manuscript. Correspondence concerning this article should be addressed to K. Arbuthnott, Campion College, University of Regina, Regina, SK, S4S 0A2 Canada (e-mail: katherine.arbuthnott@uregina.ca).

\section{REFERENCES}

Allport, D. A., Styles, E. A., \& Hsieh, S. (1994). Shifting intentional set: Exploring the dynamic control of tasks. In C. Umiltà \& M. Moscovitch (Eds.), Attention and performance XV: Conscious and nonconscious information processing (pp. 421-452). Cambridge, MA: MIT Press.

Arbuthnott, K. D. (2005). The influence of cue type on backward inhibition. Journal of Experimental Psychology: Learning, Memory, \& Cognition, 31, 1030-1042.

Arbuthnott, K. D. (in press). Asymmetric switch cost and backward inhibition: Carryover activation and inhibition in switching between tasks of unequal difficulty. Canadian Journal of Experimental Psychology.

Arbuthnott, K. [D.], \& Frank, J. [E.] (2000). Executive control in set switching: Residual switch cost and task-set inhibition. Canadian Journal of Experimental Psychology, 54, 33-41.

ArbuthnotT, K. D., \& Woodward, T. S. (2002). The influence of cuetask association and location on switch cost and alternating-switch cost. Canadian Journal of Experimental Psychology, 56, 18-29.

GADE, M., \& KoCH, I. (2005). Linking inhibition to activation in the control of task sequences. Psychonomic Bulletin \& Review, 12, 530-534.

GADE, M., \& КосH, I. (in press). Cue-independence of task inhibition: Evidence from a 2:1 cue-to-task mapping study. Canadian Journal of Experimental Psychology.
Hübner, M., Dreisbach, G., Haider, H., \& Kluwe, R. H. (2003). Backward inhibition as a means of sequential task-set control: Evidence for reduction of task competition. Journal of Experimental Psychology: Learning, Memory, \& Cognition, 29, 289-297.

Jersild, A. (1927). Mental set and shift. Archives of Psychology, 89, 5-82.

Koch, I., Gade, M., \& Philipp, A. M. (2004). Inhibition of response mode in task switching. Experimental Psychology, 51, 52-58.

Koch, I., PhILIPP, A. M., \& GADE, M. (2006). Chunking in task sequences modulates task inhibition. Psychological Science, 17, 346-350.

LogAN, G. D., \& Bundesen, C. (2003). Clever homunculus: Is there an endogenous act of control in the explicit task-cuing paradigm? Journal of Experimental Psychology: Human Perception \& Performance, 29, 575-599.

MAYr, U. (2002). Inhibition of action rules. Psychonomic Bulletin \& Review, 9, 93-99.

MAYR, U., \& BRYCK, R. L. (2007). Outsourcing control to the environment: Effects of stimulus/response locations on task selection. Psychological Research, 71, 107-116.

MAYR, U., \& KeELE, S. (2000). Changing internal constraints on action: The role of backward inhibition. Journal of Experimental Psychology: General, 129, 4-26.

MaYR, U., \& KLIEgL, R. (2000). Task-set switching and long-term memory retrieval. Journal of Experimental Psychology: Learning, Memory, \& Cognition, 26, 1124-1140.

MAYR, U., \& KLIEGL, R. (2003). Differential effects of cue changes and task changes on task-set selection costs. Journal of Experimental Psychology: Learning, Memory, \& Cognition, 29, 363-372.

PhilipP, A. M., GADE, M., \& KoCH, I. (2007). Inhibitory processes in language switching: Evidence from switching language-defined response sets. European Journal of Cognitive Psychology, 19, 395-416.

PhILIPP, A. M., \& КосH, I. (2006). Task inhibition and task repetition in task switching. European Journal of Cognitive Psychology, 18, 624-639.

Rogers, R., \& Monsell, S. (1995). Costs of a predictable switch between simple cognitive tasks. Journal of Experimental Psychology: General, 124, 207-231.

SCHNEIDER, D. W. (2007). Task-set inhibition in chunked task sequences. Psychonomic Bulletin \& Review, 14, 970-976.

$\mathrm{SCHUCH}$, S., \& KосH, I. (2003). The role of response selection for inhibition of task sets in task shifting. Journal of Experimental Psychology: Human Perception \& Performance, 29, 92-105.

Spector, A., \& Biederman, I. (1976). Mental set and mental shift revisited. American Journal of Psychology, 89, 669-679.

\section{NOTES}

1. This effect has also been labeled $n-2$ repetition cost (Koch, Philipp, $\&$ Gade, 2006) or alternating-switch cost (Arbuthnott \& Frank, 2000).

2 . These values do not add to $100 \%$, due to rounding errors for each switch condition. For each participant, the exact number of trials in each condition varied slightly.

3. Although the interaction for error rates was not significant, error rates were included in these analyses for completeness.

(Manuscript received June 25, 2007; revision accepted for publication September 19, 2007.) 